\title{
Generalized Autocalibrating Partially Parallel Acquisitions (GRAPPA)
}

\author{
Mark A. Griswold, ${ }^{1 *}$ Peter M. Jakob, ${ }^{1}$ Robin M. Heidemann, ${ }^{1}$ Mathias Nittka, ${ }^{2}$ \\ Vladimir Jellus, ${ }^{2}$ Jianmin Wang, ${ }^{2}$ Berthold Kiefer, ${ }^{2}$ and Axel Haase ${ }^{1}$
}

\begin{abstract}
In this study, a novel partially parallel acquisition (PPA) method is presented which can be used to accelerate image acquisition using an RF coil array for spatial encoding. This technique, GeneRalized Autocalibrating Partially Parallel Acquisitions (GRAPPA) is an extension of both the PILS and VD-AUTOSMASH reconstruction techniques. As in those previous methods, a detailed, highly accurate RF field map is not needed prior to reconstruction in GRAPPA. This information is obtained from several $k$-space lines which are acquired in addition to the normal image acquisition. As in PILS, the GRAPPA reconstruction algorithm provides unaliased images from each component coil prior to image combination. This results in even higher SNR and better image quality since the steps of image reconstruction and image combination are performed in separate steps. After introducing the GRAPPA technique, primary focus is given to issues related to the practical implementation of GRAPPA, including the reconstruction algorithm as well as analysis of SNR in the resulting images. Finally, in vivo GRAPPA images are shown which demonstrate the utility of the technique. Magn Reson Med 47:1202-1210, 2002. (c) 2002 Wiley-Liss, Inc.

Key words: parallel imaging; rapid MRI; RF coil arrays; SMASH; SENSE; PILS
\end{abstract}

Since the development of the NMR phased array (1) in the late 1980s, multicoil arrays have been designed to image almost every part of the human anatomy. These multicoil arrays are primarily used for their increased signal-to-noise ratio (SNR) compared to volume coils or large surface coils.

Recently, several partially parallel acquisition (PPA) strategies have been proposed (2-19) which have the potential to revolutionize the field of fast MR imaging. These techniques use spatial information contained in the component coils of an array to partially replace spatial encoding which would normally be performed using gradients, thereby reducing imaging time. In a typical PPA acquisition, only a fraction of the phase encoding lines are acquired compared to the conventional acquisition. A specialized reconstruction is then applied to the data to reconstruct the missing information, resulting in the full FOV image in a fraction of the time.

The primary limiting factor of the majority of PPA techniques is their requirement of accurate knowledge of the complex sensitivities of component coils. In practice, the

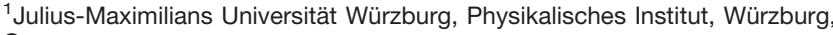
Germany.

${ }^{2}$ Siemens Medical Solutions, Erlangen, Germany.

${ }^{\star}$ Correspondence to: Mark Griswold, Department of Physics, University of Würzburg, Am Hubland, 97074 Würzburg, Germany. E-mail: mark@physik. uni-wuerzburg.de

Received 7 September 2001; revised 14 January 2002; accepted 13 February 2002.

DOI 10.1002/mrm.10171

Published online in Wiley InterScience (www.interscience.wiley.com).

(C) 2002 Wiley-Liss, Inc. actual coil sensitivity information is difficult to determine experimentally due to contamination by, for example, noise. Additionally, subject or coil motion between the time of coil calibration and image acquisition can be problematic if this information is not taken into account during the reconstruction.

Last year, we presented the parallel imaging with localized sensitivities (PILS) technique (14) and demonstrated several advantages. In PILS it is assumed that each component coil has a localized sensitivity profile. Whenever this is true, uncombined coil images can be formed for each component coil using only knowledge of the position of the coil in the FOV, which can be obtained trivially using a number of methods (14). Besides providing an efficient reconstruction process $(14,20)$, PILS was shown to provide optimal SNR for all accelerations tested, since the uncombined coil images can be combined using a sum of squares or other optimal array reconstruction method.

The PILS technique represents a departure in reconstruction philosophy compared to other previous PPA methods. In all previous methods the steps of image reconstruction (unaliasing) and SNR optimization (image combination) occur in one step. Therefore, both processes had to be simultaneously optimized for a good reconstruction. On the other hand, the process of unaliasing and SNR optimization are completely decoupled in PILS, so that both can potentially be optimized separately. We believe that this general philosophy could lead to more robust and optimized PPA reconstructions. However, in order to decouple these processes uncombined images need to be formed by the PPA reconstruction technique. To date, no such method other than PILS exists.

In this study, we describe the first extension of this basic philosophy which is applicable to coils which are not necessarily spatially localized. In this technique, GeneRalized Autocalibrating Partially Parallel Acquisitions (GRAPPA), a more generalized view of the variable density AUTO-SMASH (VD-AUTO-SMASH) technique (15), is used to generate uncombined coil images from each coil in the array. It is shown that this reconstruction process results in images in higher SNR and better overall image quality compared to previous VD-AUTO-SMASH implementations. After introduction of the reconstruction method, computer simulations will be presented which highlight the benefits of the GRAPPA reconstruction. Finally, the first in vivo results using an eight-channel receiver system and the GRAPPA reconstruction are shown.

\section{THEORY}

Review of AUTO-SMASH and VD-AUTO-SMASH

In order to understand the GRAPPA process, it is instructive to review the basics of both the AUTO-SMASH (11) 

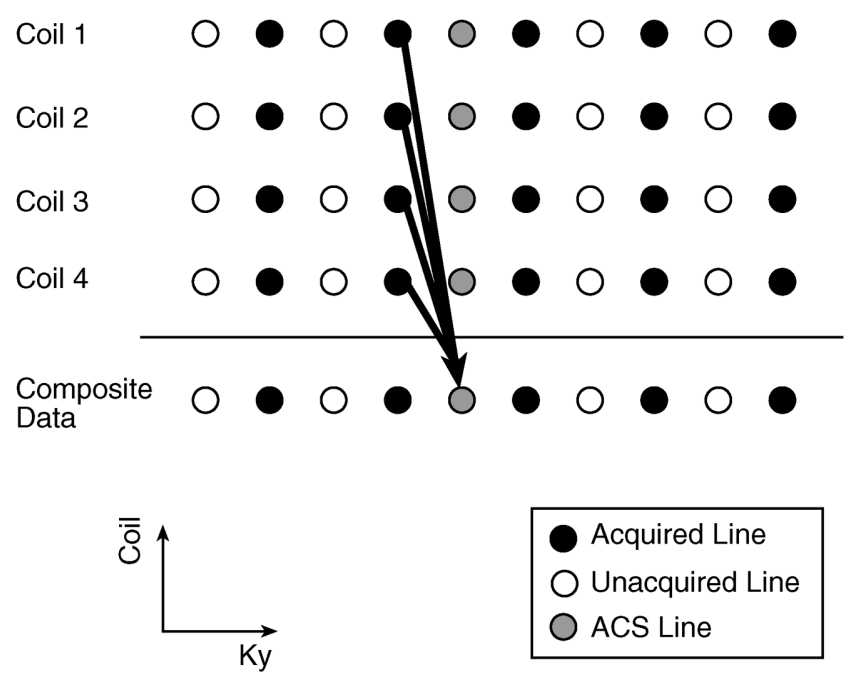

FIG. 1. The basic reconstruction although for both AUTO-SMASH and VD-AUTO-SMASH. Each circle represents a line of acquired data in a single coil. The read-out direction is left to right. In both methods a single line of data acquired in each coil in the array is fit to an ACS line in the composite image. In VD-AUTO-SMASH this process is repeated several times and the results averaged together to form the final reconstruction weights.

and VD-AUTO-SMASH (15) methods. In AUTO-SMASH, an additional calibration line, or auto-calibration signal (ACS), is collected along with the reduced data acquisition. To determine the weights used for reconstruction, a line in the reduced dataset is fit to the ACS line. This process can be represented by:

$$
\sum_{l=1}^{L} S_{l}^{A C S}\left(k_{y}-m \Delta k_{y}\right)=\sum_{l=1}^{L} n(l, m) S_{l}\left(k_{y}\right)
$$

for a line offset by $m \Delta k_{y}$, where $l$ counts through the coils up to the total number of coils, $L$. This equation is equivalent to eq. 7 in Ref. 11. The weights $n(l, m)$ are then used to reconstruct the missing lines as in the original SMASH technique (2).

This process was later extended in the VD-AUTOSMASH approach. In this method, more than one ACS line is acquired in the center of $k$-space, which allows for multiple fits to be performed for each missing line, thereby moderating the effects of both noise and coil profile imperfections (15). In addition to the improved fit provided by the VD-AUTO-SMASH approach, the extra ACS lines could be included in the final image, thereby further reducing image artifacts.

It is important to note that in both AUTO-SMASH and VD-AUTO-SMASH, as well as the original SMASH technique, the fitting process determines the weights which transform a single line acquired in each of the individual coils into a single shifted line in the composite $k$-space matrix. This process is shown schematically in Fig. 1. The data acquired in each coil (black circles) are fit to the ACS line in a composite image (gray circle), which in most cases is the simple sum of the ACS lines acquired in each coil, although in some cases the lines are phased prior to summation (13). This process is simply repeated with multiple lines and combinations in VD-AUTO-SMASH.

This process leads to two major problems found in all previous $k$-space-based reconstructions. First, the resulting SNR is that of a complex sum image (4), instead of the better sum of squares image or an image combined using optimal reconstruction weights (1). As shown previously, this can lead to losses of $\sqrt{L}$ for $L$ coils or worse at low acceleration factors. This is particularly problematic when one considers increasing the number of coils used in the reconstruction.

In addition to these SNR issues, previous $k$-space-based methods suffered from severe phase cancellation in cases where the phases of the different coils were not exactly aligned or cases where slight phase differences existed between residual aliased signal and the unaliased normal signal. In these cases, signal losses or even complete cancellation could be observed using VD-AUTO-SMASH, for example. The only way to measure this phase without collecting a complete coil map (which would defeat the advantage of any auto-calibrating approach) is to make a separate measurement of the noise correlation between the coils. However, this method can fail to provide adequate definition of the coil phases in many instances, resulting in suboptimal image quality and/or severe phase cancellation artifacts. For this reason, in previous AUTOSMASH studies (e.g. Ref. 3), great care was taken to ensure that the phases of the coils were phase-aligned prior to reconstruction.

An additional problem of these previous approaches is the potential of a poor fit of the measured lines to the ACS lines, due to the nonoptimal coil sensitivities that are encountered in practice. When this occurs, image artifacts can result for particular coil-slice orientations. While incorporating the ACS lines into the final image, as in VDAUTO-SMASH, improves the situation, residual artifact can still be a problem with certain coil geometries.

\section{GeneRalized Autocalibrating Partially Parallel Acquisitions (GRAPPA)}

GRAPPA can be seen at its most basic level as a more generalized implementation of the VD-AUTO-SMASH approach (12,15,17-19). In GRAPPA, uncombined images are generated for each coil in the array by applying multiple blockwise reconstructions to generate the missing lines for each coil. This process is shown in Fig. 2. Again, data acquired in each coil of the array (black circles) are fit to the ACS line (gray circles). However, as can be seen, data from multiple lines from all coils are used to fit an ACS line in a single coil, in this case an ACS line from coil 4. This fit gives the weights which can then be used to generate the missing lines from that coil. Once all of the lines are reconstructed for a particular coil, a Fourier transform can be used to generate the uncombined image for that coil. Once this process is repeated for each coil of the array, the full set of uncombined images can be obtained, which can then be combined using a normal sum of squares reconstruction.

In general, the process of reconstructing data in coil $j$ at a line $\left(k_{y}-m \Delta k_{y}\right)$ offset from the normally acquired data using a blockwise reconstruction can be represented by: 

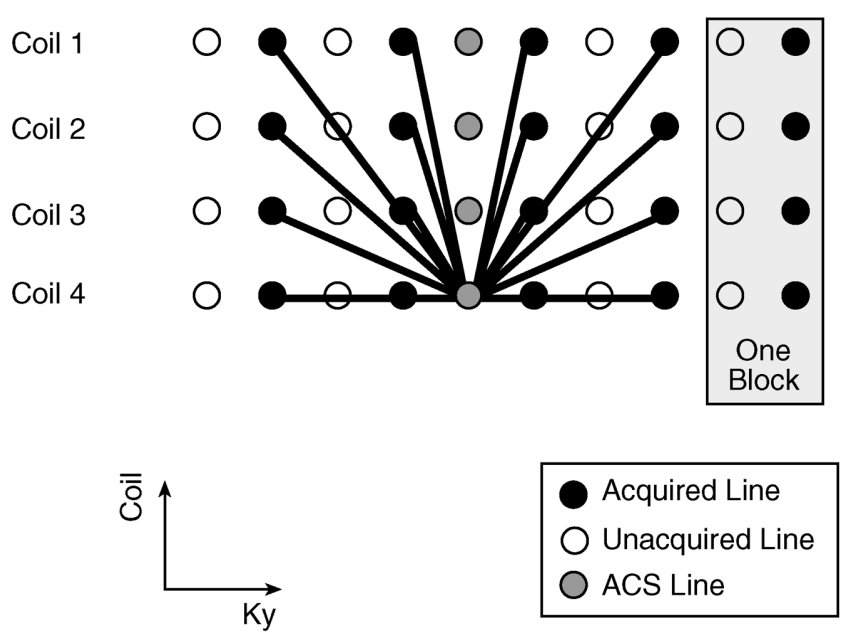

FIG. 2. The basic GRAPPA algorithm is shown schematically. In GRAPPA, more than one line acquired in each of the coils in the array are fit to an ACS line acquired in a single coil of the array. In this case, four acquired lines are used to fit a single ACS line in coil \#4. In GRAPPA, a block is defined as a single acquired line plus the missing lines adjacent to that line, as shown on the right for an acceleration factor of two.

$$
S_{j}\left(k_{y}-m \Delta k_{y}\right)=\sum_{l=1}^{L} \sum_{b=0}^{N_{b}-1} n(j, b, l, m) S_{l}\left(k_{y}-b A \Delta k_{y}\right)
$$

where $A$ represents the acceleration factor. $N_{b}$ is the number of blocks used in the reconstruction, where a block is defined as a single acquired line and $A-1$ missing lines (see Fig. 2, right side). In this case, $n(j, b, l, m)$ represents the weights used in this now expanded linear combination. In this linear combination, the index $l$ counts through the individual coils, while the index $b$ counts through the individual reconstruction blocks. This process is repeated for each coil in the array, resulting in $L$ uncombined single coil images which can then be combined using a conventional sum of squares reconstruction (1) or any other optimal array combination (21).

The use of more than one block in Eq. [2] extends the types of coil arrays and imaging planes which can be used with GRAPPA compared to previous reconstructions. The underlying assumption in AUTO-SMASH-type (and SMASH-type) reconstructions was that a perfect spatial harmonic could be generated using only the pure spatial sensitivity profiles of the coils. While this is achievable using special array geometries (22), it is not true in general, resulting in lower-quality reconstructions. By using more blocks of data to fit each missing line, GRAPPA incorporates more information into each reconstructed line, resulting in a substantially improved fit.

Using all acquired blocks for the reconstruction would result in an exact, SENSE-like reconstruction of a single coil data matrix in the absence of noise $(12,17-19)$. However, in most cases the combination in Eq. [2] can be significantly truncated to only a few blocks of lines since, in general, only lines close to each missing line contribute significant information, resulting in a small number of significant terms in Eq. [2]. For example, Sodickson (12) showed reasonable results using around seven blocks in an extended SMASH-like reconstruction.

In our experience, we have found that reconstructions using various numbers of blocks from four to eight result in essentially the same reconstruction quality. This truncation of the number of terms results in a more stable reconstruction in the presence of noise compared to an exact reconstruction using all acquired blocks in each reconstruction. Additionally, a truncated linear combination offers a more computationally efficient, yet still high quality, reconstruction, as shown below.

In order to perform the reconstruction in Eq. [2], one needs to determine the weights used in the reconstruction. As in VD-AUTO-SMASH, a block of extra ACS lines is acquired in the center of $k$-space and used to determine these complex weights $n(j, b, l, m)$ by using several fits of the form of Eq. [2]. As in VD-AUTO-SMASH, these extra acquired lines can be included in the final image reconstruction to further improve image quality (15).

The blockwise reconstruction given above has been implemented using a sliding block approach. This uses the fact that each unacquired line can be reconstructed in several different ways in a blockwise reconstruction, instead of the only one combination possible in a strictly VD-AUTO-SMASH acquisition. For example, when using four blocks for the reconstruction (i.e., four acquired lines used to reconstruct each missing line), there are four possible reconstructions for each unacquired line, two of which are shown in Fig. 3. In GRAPPA, each possible reconstruction is performed for each unacquired line, resulting in multiple possible reconstructions for each line. These lines are then combined in a weighted average to form the final reconstructed line, providing a robust recon-

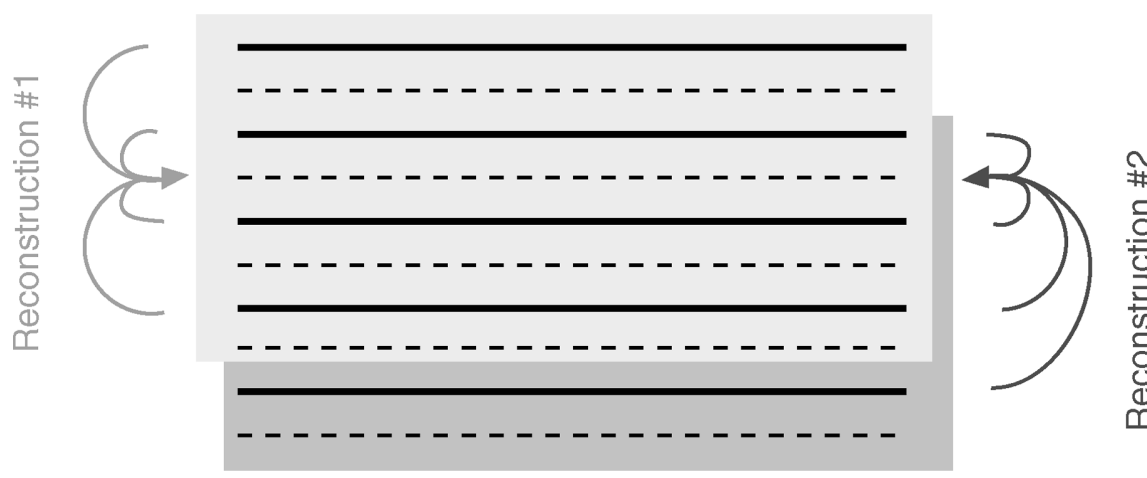

FIG. 3. In a sliding block reconstruction, more than one reconstruction is possible for each missing line. Two of the four possible reconstructions for this missing line are shown. 
struction of each missing line. For perspective, it should be noted that this sliding block reconstruction is essentially reduced to the VD-AUTO-SMASH approach whenever the number of blocks is reduced to one.

The sliding block approach introduces the problem of how to combine or optimally choose from the different reconstructions. In the images shown throughout this article, a weighted average is used to obtain the final reconstruction. Several different possibilities for performing this weighted average of the different sliding block reconstructions were tested, including the goodness of fit for a particular reconstructed line and the SNR resulting from the reconstruction of the line, given by the normalization of the weights used for each particular reconstruction (4).

While both of these criteria work in practice, the latter is preferred and used throughout this study, since in most cases where the SNR is poor for a given line the fit is also typically poor. Therefore, this method of weighting gives a reconstruction which attempts to produce both high SNR and low artifacts and has been shown to provide good results.

As demonstrated in the rest of this study, this process essentially removes the previous limitations on $k$-spacebased techniques, namely the low SNR, phase cancellation problems, and poor reconstruction quality. Since the final image combination in GRAPPA is performed using a magnitude reconstruction, any phase-related issues are essentially eliminated. No phasing information is required. Since the GRAPPA reconstruction uses a sum of squares reconstruction, the SNR efficiency of GRAPPA approaches the SNR efficiency of a sum of squares reconstruction, which can be up to $\sqrt{L}$ higher compared to previous implementations.

Finally, as has been shown previously by others $(12,17-$ 19), an approach using an extended blockwise reconstruction can lead to dramatic reductions in image artifact power compared to previous implementations. An additional benefit of the autocalibrating process used in GRAPPA is that, besides using the ACS lines to determine the coil-weighting factors, these extra lines can be integrated directly into the final image reconstructions to improve image quality, as in VD-AUTO-SMASH. As suggested by the previous studies on VD-AUTO-SMASH, it is anticipated that the use of the maximum acceleration possible in the outer parts of $k$-space is the most beneficial, since it results in the highest number of lines in the center of $k$-space for the same number of acquired lines. It has been shown that this strategy results in both a better determination of the coil-weighting factors in the presence of noise and a more robust reconstruction in the presence of imperfect coil performance (15).

\section{MATERIALS AND METHODS}

The GRAPPA reconstruction algorithm as described in the previous section was implemented in the Matlab programming environment (MathWorks, Natick, MA). As implemented, the user is free to decide the number of blocks to use in each reconstruction. As mentioned above, in our experience the numbers of blocks in the range of four to eight showed similar good results. Therefore, four blocks were used in each reconstruction for simplicity and to minimize computational complexity of each reconstruction; however, as mentioned above, images with more blocks showed essentially the same image quality.

\section{Computer Simulations}

Computer simulations were performed to establish the SNR performance of GRAPPA at different acceleration factors for a typical imaging array. To this end, the fourelement linear array found in Ref. 22 was simulated using an analytic integration of the Biot-Savart equation. These coil sensitivities were then used with an image from a standard resolution phantom to generate several datasets with reduced encoding to determine the SNR performance of the GRAPPA technique at various acceleration factors. These calculations were performed for both the VD-AUTOSMASH reconstruction which results in a complex sum reconstruction and a GRAPPA reconstruction using a sum of squares reconstruction to demonstrate the benefits of the uncombined-coil approach. SNR was estimated using multiple reconstructions of a phantom image each with different additive noise. The SNR on a pixel-by-pixel basis can then be calculated as the mean of the signal at each pixel divided by the standard deviation at that pixel over the various reconstructed images.

\section{In Vivo Imaging}

Several different imaging studies were performed on healthy volunteers to assess the clinical application of the GRAPPA technique. Informed consent was obtained before each study. Volunteers were scanned using a $1.5 \mathrm{~T}$ Siemens Quantum Symphony scanner (maximum gradient strength: $30 \mathrm{mT} / \mathrm{m}$, slew rate: $125 \mathrm{~T} / \mathrm{m} / \mathrm{s}$ ) equipped with eight receiver channels using either the standard fourelement body array or a prototype eight-element cardiac array (Siemens Medical Systems, Erlangen, Germany). This prototype array consists of eight rectangular coils $(200 \times 120 \mathrm{~mm})$ overlapped to null the mutual inductance between neighboring elements, resulting in a total width in the left-right direction of $444 \mathrm{~mm}$. Four elements are contained in a rigid frame which is placed on the scanner bed, while the upper four elements are flexible and were bent to fit each volunteer individually.

As an example of the improvement that can be obtained using GRAPPA for conventional imaging, a 3D axial gradient echo acquisition (FOV $300 \times 400 \mathrm{~mm}$, matrix $127 \times$ 256, TR $6.7 \mathrm{~ms}$, TE $3.2 \mathrm{~ms}$, flip angle $12^{\circ}, 44$ partitions each $4 \mathrm{~mm}$ thick) was used in combination with the standard four-channel body array. For this acquisition, only a full dataset was acquired and later decimated off-line by a factor of two to simulate a reduced acquisition. This dataset was then reconstructed using both the PILS reconstruction (14) and the GRAPPA reconstruction for comparison of image quality. For the coil position information in PILS, a reference line derived from a projection through the body was used. For the GRAPPA reconstruction, 16 lines were used to solve for the reconstruction parameters, although none of these extra reference lines were used to simulate variable density sampling in the final image.

As a further example, a segmented TrueFISP sequence was used for cardiac cine imaging on a healthy volunteer in combination with the eight-element prototype array. 


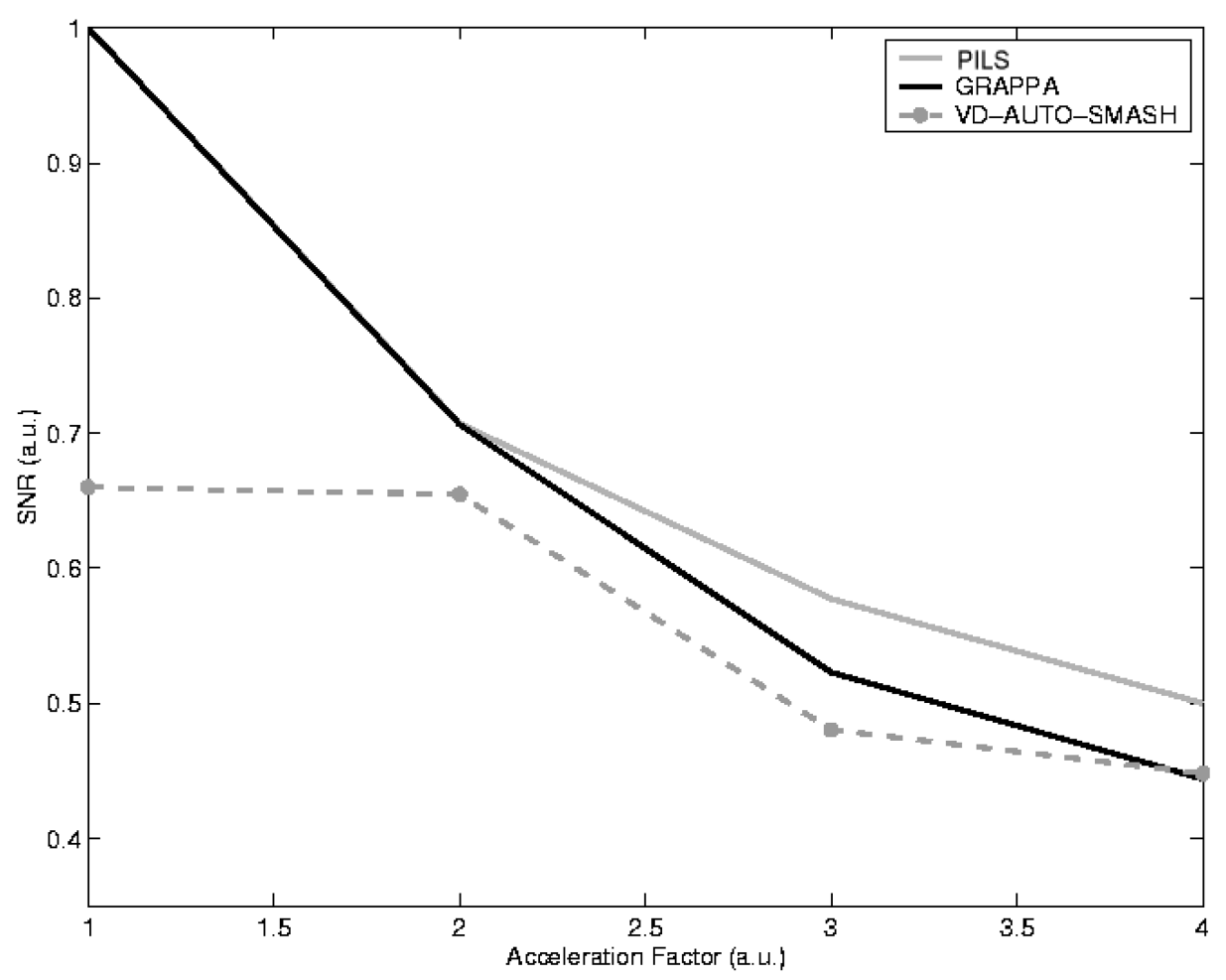

FIG. 4. SNR vs. acceleration factor for GRAPPA with uncombined coil reconstruction, VD-AUTO-SMASH reconstruction, and a PILS reconstruction. GRAPPA has substantially higher SNR than the VD-AUTOSMASH reconstruction, in particular at lower accelerations.
The sequence parameters were $\mathrm{TR}=3.64 \mathrm{~ms}$, $\mathrm{TE}=$ $1.82 \mathrm{~ms}, 15$ lines/segment, FOV $=276 \times 340 \mathrm{~mm}$, matrix $210 \times 256$, slice thickness $=6 \mathrm{~mm}$, flip angle $=70^{\circ}$. Using the GRAPPA approach with an acceleration factor of two, 18 phases could be acquired over nine heartbeats. In this case, eight additional ACS lines were acquired during the first phase of the cardiac cycle and the weights calculated from these lines were used for all other phases. This sequence was repeated in another volunteer and was reconstructed with both the AUTO-SMASH and VD-AUTOSMASH techniques to demonstrate the benefits of GRAPPA over the previous techniques.

Besides traditional anatomical imaging, we investigated the performance of GRAPPA in single-shot acquisitions. Unlike multishot experiments, these techniques are affected by spin relaxation and evolution over the entire course of an acquisition. In these cases, PPA techniques have previously been shown to improve image resolution in all single-shot imaging sequences and to decrease image distortions in EPI (23).

However, in single-shot imaging situations, a variable density sampling approach cannot be used, since fully sampling lines in the center of $k$-space would result in a decrease in image quality. Therefore, in all single-shot experiments, a separate acquisition of ACS lines was applied before the acquisition of the reduced encoded image data. These lines were then only used for determination of the coil-weighting factors and then discarded.

To study the benefits of combining GRAPPA with singleshot imaging in the abdomen and chest, several sequences were used. A multislice HASTE was used to image both the liver and kidneys using the prototype cardiac array. An inversion recovery HASTE was used to image the lung, also with the cardiac array. The sequence parameters used in each of these acquisitions is given in their respective figures.

\section{RESULTS}

\section{Computer Simulations}

The results of the computer simulations are shown in Fig. 4 and Table 1. As can be seen, the GRAPPA reconstruction using the sum of squares reconstruction results in higher SNR than the VD-AUTO-SMASH complex sum approach at all accelerations tested. For this particular coil arrangement and slice orientation, the GRAPPA approach with the sum of squares reconstruction achieves nearly perfect SNR efficiency at the acceleration factors tested. It should also be noted that in the limit where all lines are acquired, GRAPPA approaches the sum of squares reconstruction, so that nearly no loss in performance should be expected, especially for low acceleration factors.

\section{In Vivo Imaging}

Figure 5 shows a comparison between the GRAPPA reconstruction and the PILS reconstruction using the same basic

Table 1

SNR vs. Acceleration Factor for GRAPPA and VD-AUTO-SMASH

\begin{tabular}{|c|c|c|c|c|}
\hline SNR & $1 \mathrm{X}$ & $2 X$ & $3 X$ & $4 X$ \\
\hline Relative SNR GRAPPA & 1.000 & 0.706 & 0.522 & 0.443 \\
\hline Relative SNR/Time GRAPPA & 1.000 & 0.999 & 0.905 & 0.887 \\
\hline Relative SNR- & & & & \\
\hline VD-AUTO-SMASH & 0.660 & 0.654 & 0.480 & 0.448 \\
\hline Relative SNR/Time- & & & & \\
\hline VD-AUTO-SMASH & 0.660 & 0.926 & 0.832 & 0.896 \\
\hline
\end{tabular}



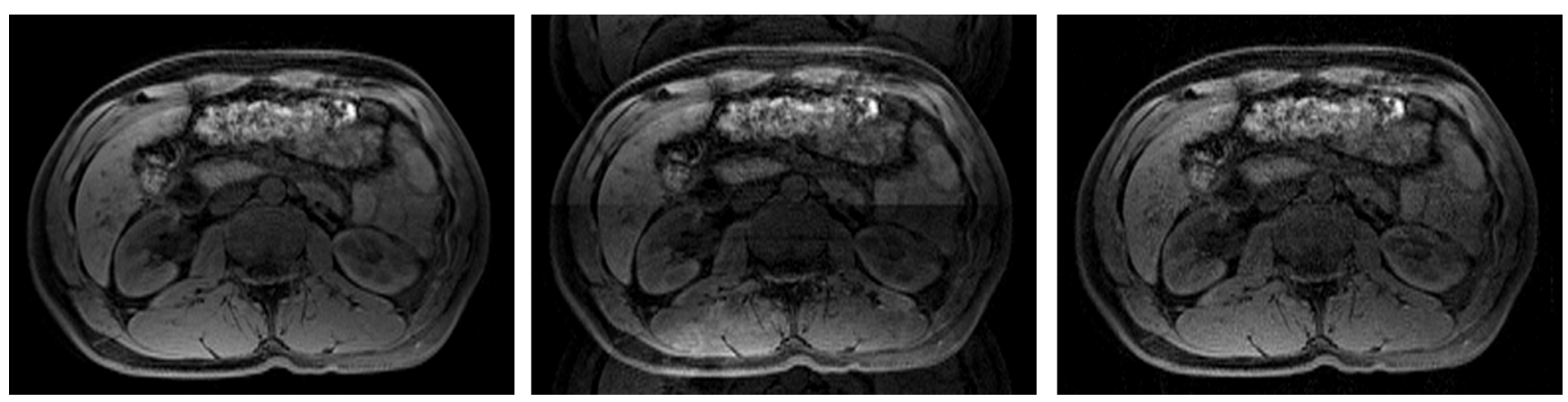

FIG. 5. Comparison of GRAPPA and PILS using a four-channel array. Left: Full time reference scan (middle) PILS reconstruction with acceleration factor two. Notice the residual aliasing as well as the significant discontinuity near the middle of the FOV due to the extended sensitivity of each coil, which in this case extends thoughout the entire sample. Right: GRAPPA reconstruction with acceleration factor of two. Besides the visible reduction in SNR, image quality is comparable to the full-time reference image.

dataset and the four-channel body array. This body array consists of a set of anterior coils and a set of posterior elements. On each side, two quadrature pairs of coils are arranged in the head-foot direction.

With this coil arrangement, the majority of the signal for any one slice in the axial imaging plane comes from only two coils of the array, so that this case represents a significant challenge for any PPA technique. As can be seen in the middle image of Fig. 5, this situation is completely unsuitable for the application of the PILS technique, since the coil sensitivities are not localized along the phaseencoding direction, and in fact have significant sensitivity across the entire width of the sample along the phaseencoding direction. This results in both residual aliasing and in a significant discontinuity in the middle of the image at the junction of the two PILS subimages. On the other hand, the GRAPPA reconstruction (Fig. 5, right) shows nearly perfect image quality, besides the loss in SNR seen when using any parallel imaging technique.

Figure 6 shows eight uncombined images acquired using the TrueFISP cine sequence at an acceleration factor of two using the eight-element cardiac array. It is clear that the extended GRAPPA approach results in images which are free of aliasing, so that each subimage in this figure is

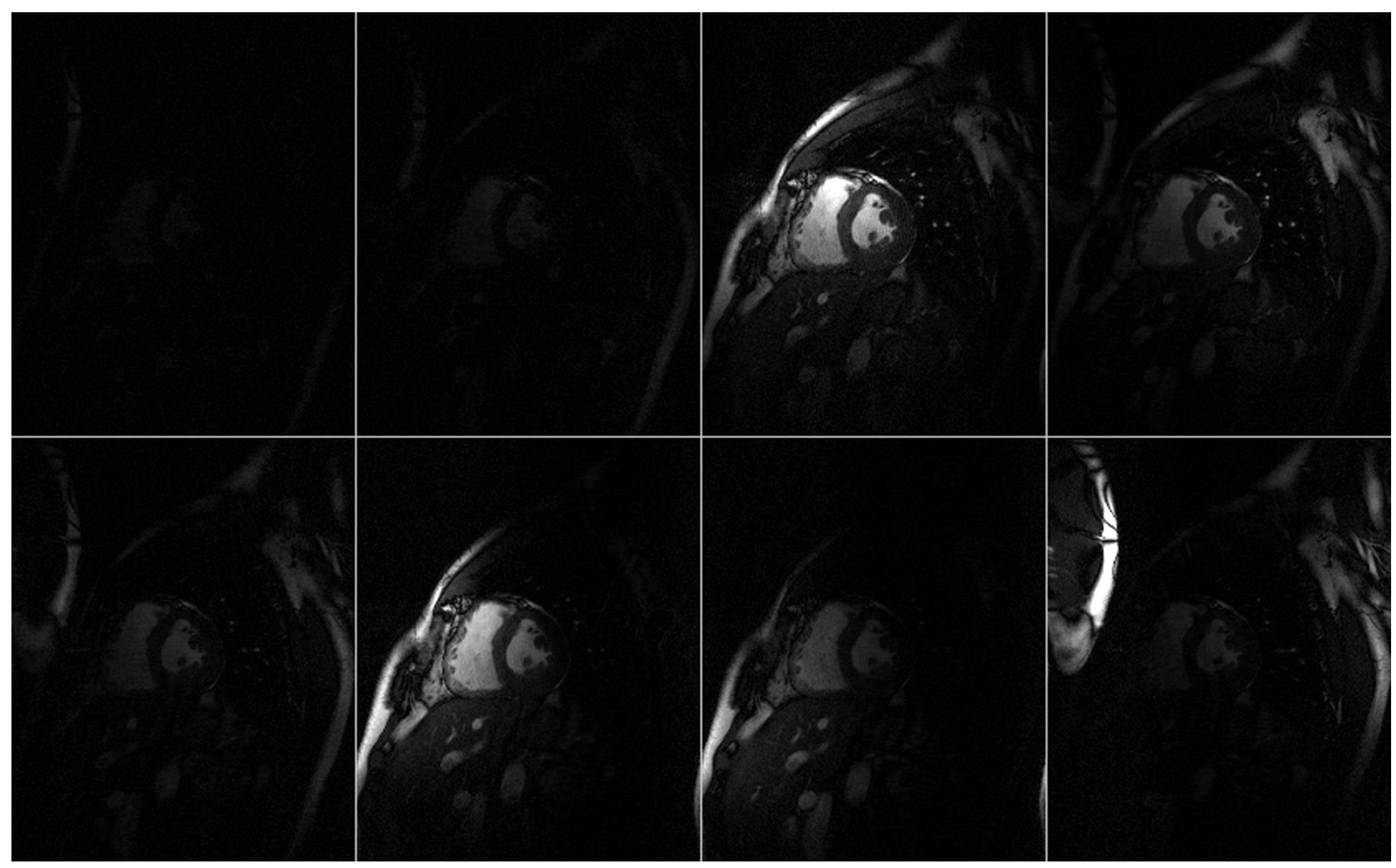

FIG. 6. Segmented TrueFISP cine images. Uncombined coil images from the GRAPPA reconstruction of a single frame of a segmented TrueFISP cine sequence. All images are shown on the same intensity scale. 

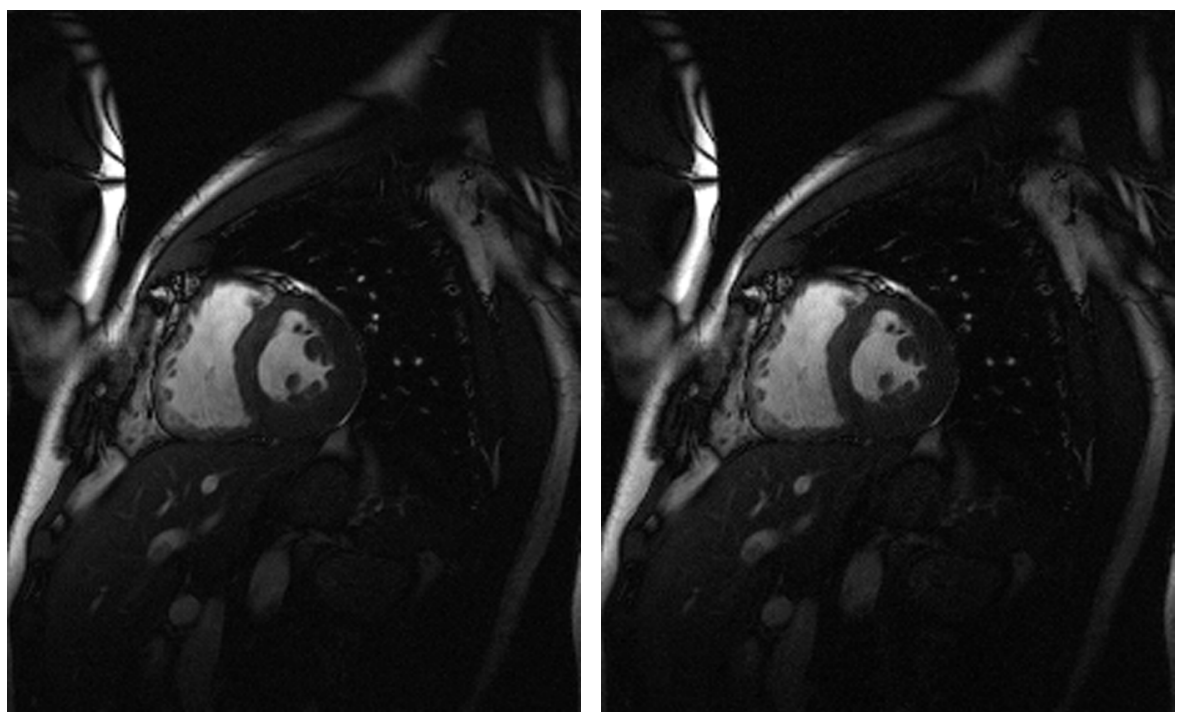

FIG. 7. Segmented TrueFISP cine images. Left: Standard full-time acquisition and sum of squares reconstruction. Right: GRAPPA reconstruction with acceleration factor of two.

essentially equivalent to the image that would have been acquired if full gradient encoding was used. It should be noted that for this imaging plane several coils (e.g., coils 1 and 2) contribute little signal. However, even in the case of low SNR the GRAPPA approach provides good reconstructions for these coil elements.

Once the uncombined images are formed they can be combined using almost any array combination algorithm. The combination using a sum of squares reconstruction is shown in Fig. 7, along with the results from a fully gradient encoded image. As can be seen, the GRAPPA image acquired nearly a factor of two faster results in essentially the same image quality as the slower fully gradient encoded image.

Figure 8 shows how the various reconstructions perform for this particular application in a different volunteer. All of the images from Fig. 8b-d were reconstructed from the same raw dataset using different reconstructions. In this case, the AUTO-SMASH reconstruction which uses a single ACS line suffers from both residual aliasing artifacts and, more importantly in this case, severe phase cancellation artifacts. The VD-AUTO-SMASH reconstruction (Fig. 8c) using eight additional lines provides an image essentially free of aliasing artifacts; however, the severe phase cancellation and the resulting low SNR renders the image diagnostically useless. On the other hand, GRAPPA (Fig. 8d) provides a nearly perfect reconstruction free of phase cancellation artifacts and with higher SNR than either of the other reconstructions.

Figure 9 demonstrates the benefit of employing the GRAPPA approach for lung imaging. For reference, the left image is the fully gradient-encoded image acquired with the minimum interecho spacing available on the system. Substantial blurring can be seen in the lung tissues, due to the relatively short $T_{2} \mathrm{~s}$ in this region. This is not the case in the three times accelerated GRAPPA image shown on the right. The GRAPPA image on the right provides a substantial gain in resolution compared to the conventionally encoded image on the left (23). The decreased effective interecho spacing and the increased matrix size combined provide a dramatic increase in the visibility of small vessels, in particular the small vessels in the lower lobes of both lungs are clearer in the GRAPPA image.

As a final example, Fig. 10 shows a typical example of a GRAPPA HASTE image in the liver. Again, the decreased effective interecho spacing and increased matrix size provide enhanced visibility of the vessels in the liver. In addition, it is clear from these images that the subcutaneous fat is considerably sharper in the GRAPPA image compared to the conventional image. This is expected
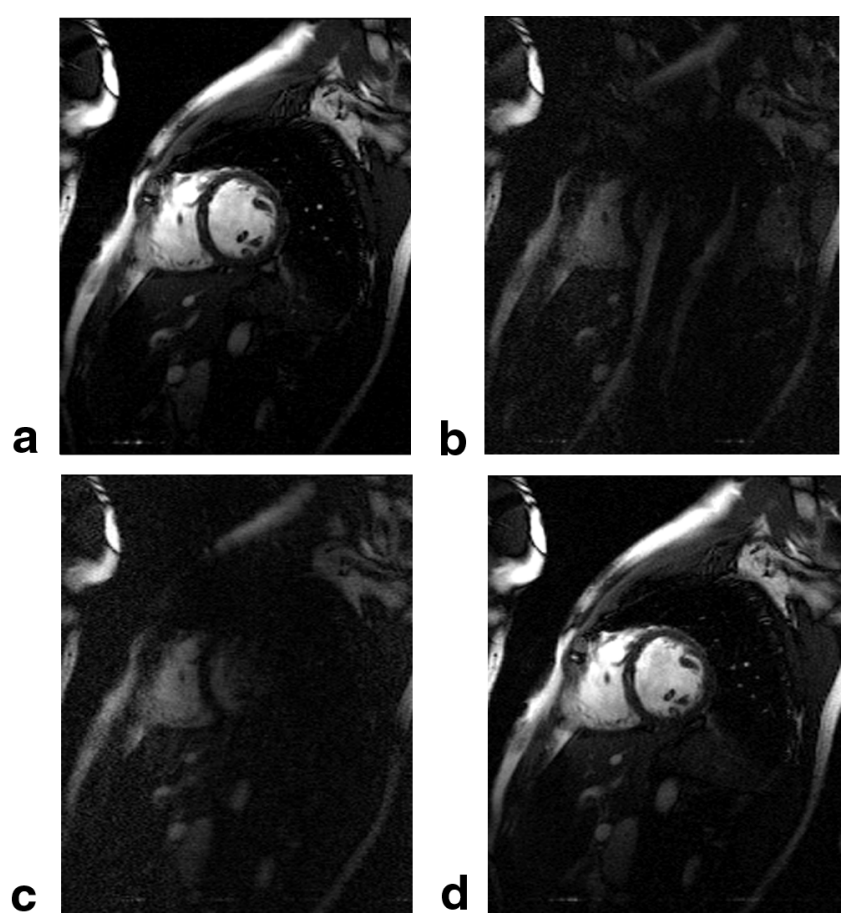

FIG. 8. TrueFISP cine results reconstructed using the various reconstruction strategies. a: Full-time reference image. b: AUTOSMASH reconstruction without phase correction of coil images. c: VD-AUTO-SMASH reconstruction with eight ACS lines without phase correction of coil images. d: GRAPPA reconstruction with eight ACS lines. 

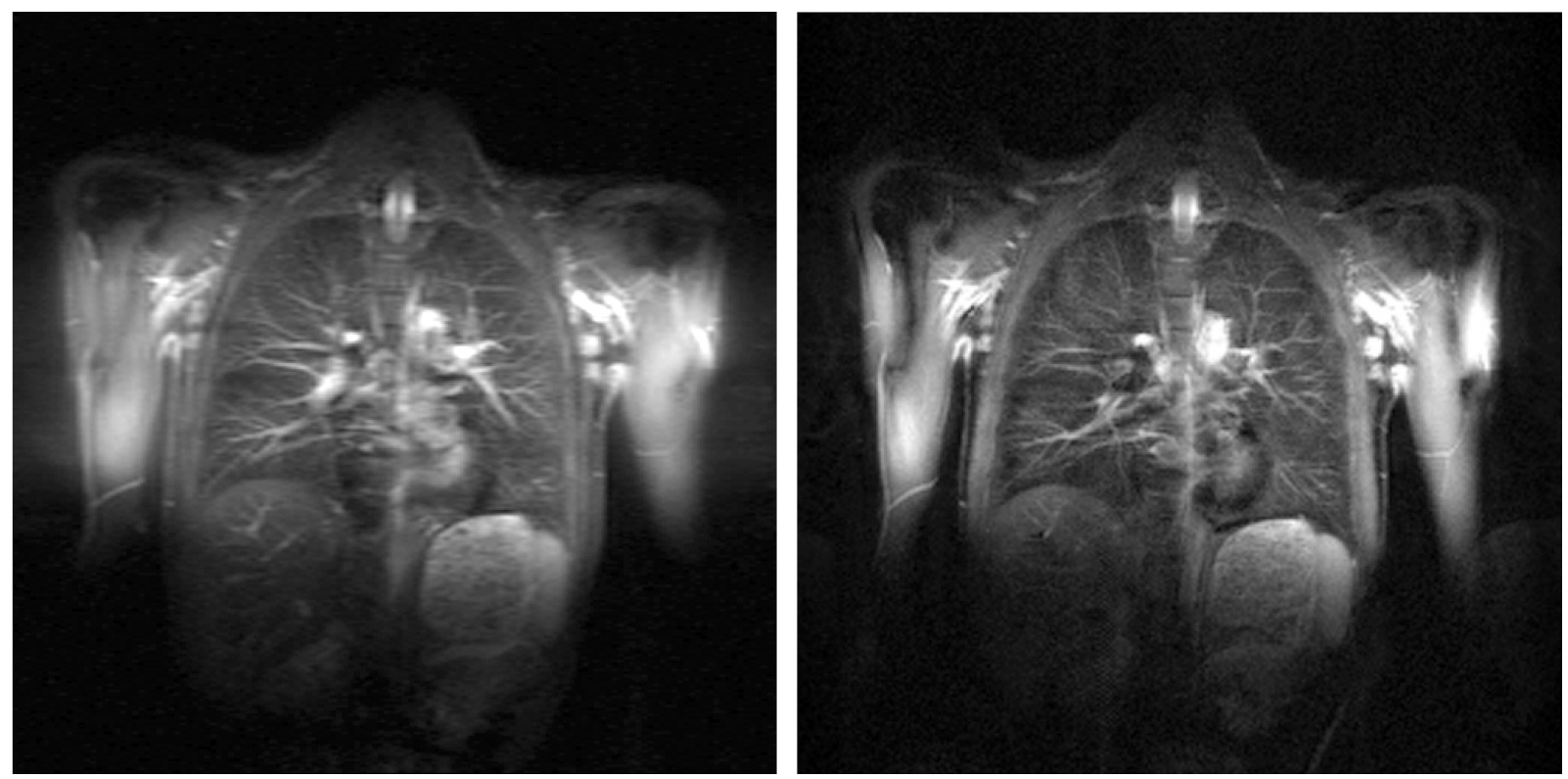

FIG. 9. Single-shot inversion recovery HASTE images in the lungs of a healthy volunteer acquired with an eight-element cardiac array. (Interecho spacing $=2.88 \mathrm{~ms}$, bandwidth $=781 \mathrm{~Hz} /$ pixel, FOV $=500 \times 500 \mathrm{~mm}$, Slice thickness $=10 \mathrm{~mm}$ ). Left: Conventional full time acquisition matrix $=128 \times 256$ (207 ms). Right: GRAPPA acquisition (acceleration factor 3) with matrix $256 \times 256$ (150 ms). In addition to the lack of any phase-related artifacts, the GRAPPA image demonstrates increased resolution and decreased blurring compared to the conventional acquisition. The phase-encoding direction is horizontal in these images.

since the $T_{2}$ relaxation time of fat is very short compared to the other tissues in this slice.

This increased resolution in fat visualization could be especially useful in liver pathologies which involve large fatty deposits in the liver, allowing potentially better diagnosis and/or delineation of disease progression.

\section{DISCUSSION}

In this study, we have introduced the basic GRAPPA concept. In this method uncombined coil images are reconstructed from the undersampled acquisitions which can then be combined using any array combination method. In this study, a sum of squares combination was used. How- ever, several other possibilities exist. As an example, the adaptive array combination method proposed by Walsh et al. (21) could be used to further improve the image quality in GRAPPA reconstructions. This method was shown by Walsh et al. to provide SNR approaching a Roemer optimal combination and decreased background noise compared to a sum of squares reconstruction. This method is particularly advantageous since it does not require any additional coil mapping information; all required information could be derived from the reconstructed images themselves.

Over the last few years, parallel imaging strategies have seen increased acceptance in the MRI community. In particular, the sensitivity encoding (SENSE) method (5) has been implemented by many international groups with very
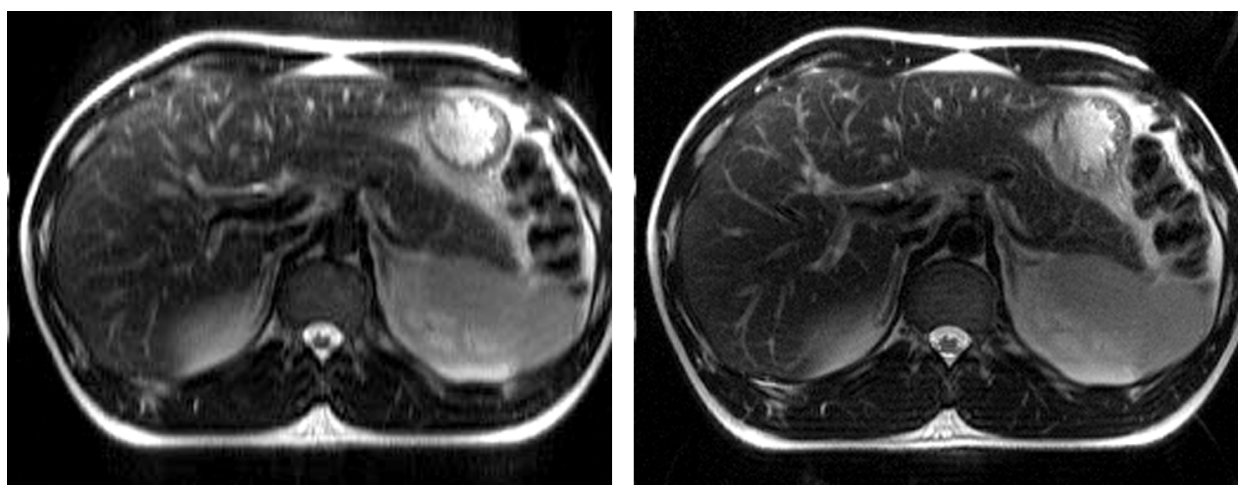

FIG. 10. Single-shot HASTE images in the liver of a healthy volunteer acquired with an eight-element cardiac array (Interecho spacing $=$ $3.5 \mathrm{~ms}$, bandwidth $=781 \mathrm{~Hz} /$ pixel, FOV $=240 \times 320 \mathrm{~mm}$, Slice thickness $=8 \mathrm{~mm}$.) Conventional full time acquisition (left) matrix $128 \times$ 256 (252 ms). GRAPPA acquisition in the same imaging time (right) matrix $256 \times 256$ (252 ms). Notice the increased resolution and decreased blurring in the GRAPPA image compared to the conventional acquisition. The phase-encoding direction is vertical in these images. 
good results. SENSE provides an optimized reconstruction whenever a perfectly accurate coil sensitivity map can be obtained. Therefore, whenever a high-quality estimate of the coil sensitivities can be obtained, SENSE should be the method of choice. However, there are clearly a number of cases where the acquired coil map may be in error, and therefore an autocalibrating technique such as GRAPPA should be used. In our experience, patient motion, including respiratory motion, can lead to substantial errors in acquired sensitivity maps, in particular at the coil edges where the coil sensitivity changes rapidly. Patient motion is not necessarily a problem in GRAPPA, since the coil sensitivity information can be collected along with the reduced phase encoding acquisition to ensure proper reconstruction. Another area where coil sensitivity maps may be in error is single-shot EPI in regions with severe distortions (24). In this case, the image intensity at a given location may not correspond to the correct value in the sensitivity map since, it could be shifted along the phaseencoding direction. Our preliminary experience with GRAPPA in the presence of severe EPI distortions has been positive (24) and will be the subject of a future publication.

Finally, as noted previously, all PPA methods are expected to benefit from increasing numbers of array elements. To our knowledge, this is the first study to combine PPA imaging with an eight-channel receiver system. In our experience, the most significant aspect of these systems with more channels is their ability to more optimally image in a variety of imaging planes. With only four elements, the range of possible slice orientations which are optimal is rather limited. However, whenever more elements can be placed around the body there is a greater chance that an optimal configuration will be present for nearly every imaging plane. While eight elements provided significant benefits over the previous four-channel systems, we anticipate further progress in this area, so that systems with 16-32 channels will become available in the near future. These systems with even more channels are expected to provide even more benefits in the area of parallel imaging.

\section{CONCLUSION}

In this study we introduced the GRAPPA parallel imaging method. This method is an extension of both the PILS and VD-AUTO-SMASH methods. An extended blockwise VDAUTO-SMASH reconstruction is used to reconstruct uncombined coil images from each coil in the array. These images can then be combined using any common array reconstruction method, such as sum of squares. In this study, good quality images were shown from cardiac, lung, and abdominal applications. The autocalibrating nature of GRAPPA is particularly interesting in areas where coil sensitivity maps may be difficult to obtain, such as cardiac and abdominal imaging, as well as single-shot EPI applications. Studies are currently ongoing to investigate the application of GRAPPA to these particular areas.

\section{REFERENCES}

1. Roemer PB, Edelstein WA, Hayes CE, Souza SP, Mueller OM. The NMR phased array. Magn Reson Med 1990;16:192-225.

2. Sodickson DK, Manning WJ. Simultaneous acquisition of spatial harmonics (SMASH): fast imaging with radiofrequency coil arrays. Magn Reson Med 1997;38:591-603.

3. Jakob PM, Griswold MA, Edelman RR, Manning WJ, Sodickson DK. Accelerated cardiac imaging using the SMASH technique. J Cardiovasc Magn Reson 1999;1:153-157.

4. Sodickson DK, Griswold MA, Jakob PM, Edelman RR, Manning WJ. Signal-to-noise ratio and signal-to-noise efficiency in SMASH imaging. Magn Reson Med 1999;41:1009-1022.

5. Pruessmann KP, Weiger M, Scheidegger MB, Boesiger P. SENSE: sensitivity encoding for fast MRI. Magn Reson Med 1999;42:952-962.

6. Kelton J, Magin RM, Wright SM. An algorithm for rapid image acquisition using multiple receiver coils. In: Proc 8th Scientific Meeting, ISMRM, 1989. p 1172.

7. Carlson JW, Minemura T. Imaging time reduction through multiple receiver data acquisition and image reconstruction. Magn Reson Med 1993;29:681-688.

8. Ra JB, Rim CY. Fast imaging using subencoding data sets from multiple detectors. Magn Reson Med 1993;30:142-145.

9. Wang J, Reykowski A. A SMASH/SENSE related method using ratios of array coil profiles. In: Proc 7th Scientific Meeting, ISMRM, Philadelphia, 1999. p 1648.

10. Weiger M, Pruessmann KP, Boesiger P. High performance cardiac realtime imaging using SENSE. In: Proc 7th Scientific Meeting, ISMRM, 1999. p 385.

11. Jakob PM, Griswold MA, Edelman RR, Sodickson DK. AUTO-SMASH: a self-calibrating technique for SMASH imaging. MAGMA 1998;7:4254.

12. Sodickson DK. A generalized basis approach to spatial encoding with coil arrays: SMASH-SENSE hybrids and improved parallel MRI at high accelerations. In: Proc 8th Scientific Meeting, ISMRM, Denver, 2000. p 273.

13. Sodickson DK. Tailored SMASH image reconstructions for robust in vivo parallel MRI. Magn Reson Med 2000;44:243-251.

14. Griswold MA, Jakob PM, Heidemann RM, Haase A. Parallel imaging with localized sensitivities (PILS). Magn Reson Med 2000;44:602-609.

15. Heidemann RM, Griswold MA, Haase A, Jakob PM. VD-AUTO-SMASH imaging. Magn Reson Med 2001;45:1066-1074.

16. Walsh DO, Gmitro AF. Sub-sampled phased array MRI reconstruction via coherent spatial nulling. In: Proc 8th Scientific Meeting, ISMRM, 2000. p 1720 .

17. Wang J. Generalized parallel imaging using array coil encoding. In: Proc 9th Scientific Meeting, ISMRM, Glasgow, 2001. p 769.

18. Bankson JA, Wright SM. Generalized partially parallel imaging with spatial filters. In: Proc 9th Scientific Meeting, ISMRM, Glasgow, 2001. p 1794.

19. Bydder M, Larkman DJ, Hajnal JV. Generalized SMASH imaging. Magn Reson Med 2002;47:160-170.

20. Eggers H, Boernert P, Boesiger P. Real-time partial parallel spiral imaging with localized sensitivities. In: Proc 9th Scientific Meeting, ISMRM, Glasgow, 2001. p 1772.

21. Walsh DO, Gmitro AF, Marcellin MW. Adaptive reconstruction of phased array MR imagery. Magn Reson Med 2000;43:682-690.

22. Griswold MA, Jakob PM, Edelman RR, Sodickson DK. An RF coil array designed specifically for cardiac SMASH imaging. MAGMA 2000;10: 105-113.

23. Griswold MA, Jakob PM, Chen Q, Goldfarb JW, Manning WJ, Edelman RR, Sodickson DK. Resolution enhancement in single shot imaging using simultaneous acquisition of spatial harmonics (SMASH). Magn Reson Med 1999;41:1236-1245.

24. Griswold MA, Jakob PM, Heidemann RM, Nittka M, Wang J, Kiefer B, Haase A. Off-resonance artifacts in single shot EPI using partially parallel imaging. In: Proc 9th Scientific Meeting, ISMRM, Glasgow, 2001. p 446. 